\title{
APOE4 Allele Disrupts Resting State fMRI Connectivity in the Absence of Amyloid Plaques or Decreased CSF A $\beta 42$
}

\author{
Yvette I. Sheline, ${ }^{1,2,3,6}$ John C. Morris, ${ }^{3,6}$ Abraham Z. Snyder, ${ }^{2}$ Joseph L. Price, ${ }^{4}$ Zhizi Yan, ${ }^{2}$ Gina D'Angelo, ${ }^{5,6}$ Collin Liu, ${ }^{2}$ \\ Sachin Dixit, ${ }^{2}$ Tammie Benzinger, ${ }^{2}$ Anne Fagan, ${ }^{3,6}$ Alison Goate, ${ }^{1,3,6}$ and Mark A. Mintun ${ }^{1,2,6}$ \\ Departments of ${ }^{1}$ Psychiatry, ${ }^{2}$ Radiology, ${ }^{3}$ Neurology, ${ }^{4}$ Anatomy and Neurobiology, and ${ }^{5}$ Biostatistics, and ${ }^{6}$ The Knight Alzheimer's Disease Research \\ Center, Washington University School of Medicine, St. Louis, Missouri 63110
}

Identifying high-risk populations is an important component of disease prevention strategies. One approach for identifying at-risk populations for Alzheimer's disease (AD) is examining neuroimaging parameters that differ between patients, including functional connections known to be disrupted within the default-mode network. We have previously shown these same disruptions in cognitively normal elderly who have amyloid- $\beta(\mathrm{A} \beta)$ plaques [detected using Pittsburgh Compound B (PIB) PET imaging], suggesting neuronal toxicity of plaques. Here we sought to determine if pathological effects of apolipoprotein $\mathrm{E} \varepsilon 4$ (APOE4) genotype could be seen independent of $\mathrm{A} \beta$ plaque toxicity by examining resting state fMRI functional connectivity (fcMRI) in participants without preclinical fibrillar amyloid deposition (PIB - ). Cognitively normal participants enrolled in longitudinal studies $(n=100$, mean age $=62)$ who were PIBwere categorized into those with and without an APOE4 allele and studied using fcMRI. APOE4 allele carriers (E4+) differed significantly from $\mathrm{E} 4$ - in functional connectivity of the precuneus to several regions previously defined as having abnormal connectivity in a group of AD participants. These effects were observed before any manifestations of cognitive changes and in the absence of brain fibrillar $\mathrm{A} \beta$ plaque deposition, suggesting that early manifestations of a genetic effect can be detected using fcMRI and that these changes may antedate the pathological effects of fibrillar amyloid plaque toxicity.

\section{Introduction}

Successfully identifying disease imaging biomarkers may contribute to understanding disease pathogenesis and establish thresholds for measuring disease progression and response to therapies. Using imaging parameters to identify how early intervention needs to occur to prevent neuronal injury in the pathological progression to Alzheimer's disease (AD) is an important component of disease prevention strategies. Imaging tracers such as Pittsburgh Compound B (PIB), which bind to fibrillar deposits of amyloid- $\beta(\mathrm{A} \beta)$, allow in vivo detection of this pathologic hallmark of $\mathrm{AD}$, although amorphous diffuse $\mathrm{A} \beta$ plaques may not be detected by PIB (Cairns et al., 2009). Brain areas, including the precuneus, constitute a network with correlated spontaneous

\footnotetext{
Received July 21, 2010; revised Sept. 23, 2010; accepted 0ct. 18, 2010.

This work was supported by National Institutes of Health Grants K24MH079510 (to Y.I.S.), P50AG05681 (to J.C.M. and Y.I.S.), P01AG03991 (to J.C.M.), P01AG026275 (to J.C.M.), P30NS048056 (to M.A.M. and A.Z.S.), and P30NS06833 (to M.A.M. and A.Z.S.). We thank all of the Washington University Alzheimer's Disease Research Center personnel for their skilled assistance and the participants for their time and effort.

Neither the granting agencies nor any other funding entity had a role in any of the following aspects of this study: design and conduct of the study; collection, management, analysis, and interpretation of the data; or preparation, review, or approval of the manuscript. Y.I.S. is independent of any commercial provider, had full access to all of the data in this study, and takes responsibility for the integrity of the data and the accuracy of the data analysis. Y.I.S. has served on the advisory board and speakers bureau of Lilly. J.C.M. has served as a consultant for the following companies: AstraZeneca, Bristol-Myers Squibb, Elan, Genentech, Lilly, Merck, Novartis, Pfizer, Schering Plough, and Wyeth. A.G. has served as a consultant for AstraZeneca. M.A.M. is now employed by Avid Radiopharmaceuticals. All other authors report no potential conflict of interest. No author named on the title page of this study has any financial interest in the results of the study nor any other conflict of interest relevant to the subject matter of this manuscript.

Correspondence should be addressed to Dr. Yvette Sheline, Department of Psychiatry, Box 8134, Washington University School of Medicine, 660 South Euclid Avenue, St. Louis, M0 63110. E-mail: yvette@npg.wustl.edu.

DOI:10.1523/JNEUROSCI.3987-10.2010

Copyright $\odot 2010$ the authors $\quad 0270-6474 / 10 / 3017035-06 \$ 15.00 / 0$
}

brain activity at rest, the default-mode network (DMN), which is more active at rest and less active during tasks (Raichle et al., 2001). DMN regions are among the regions earliest affected by amyloid deposition in $\mathrm{AD}$ (Mintun et al., 2006).

Previous work has shown that, compared with brain activity in cognitively normal individuals (Greicius and Menon, 2004), brain activity in $\mathrm{AD}$ is associated with $\mathrm{DMN}$ resting-state $\mathrm{AMRI}$ functional connectivity (fcMRI) disruptions. Because $\sim 25 \%$ of older cognitively normal individuals have fibrillar $A \beta$ deposition by PIB PET (PIB+) (Mintun et al., 2006), similar to postmortem work (Price and Morris, 1999), we recently investigated the effect of $\mathrm{A} \beta$ on DMN resting-state connectivity. We found that, in cognitively normal elderly, the presence of fibrillar amyloid $(\mathrm{PIB}+)$ was associated with regional disruptions in fcMRI of the same $\mathrm{DMN}$ regions as in $\mathrm{AD}$, including precuneus, hippocampus, parahippocampus, anterior cingulate cortex, and visual cortex (Sheline et al., 2010). Disruption in resting-state connectivity in subjects with $\mathrm{A} \beta$ plaque deposition has also been identified by other investigators (Hedden et al., 2009).

In addition to an amyloid toxicity effect in producing neuronal damage, a number of studies have demonstrated the importance of the $\varepsilon 4$ allele of apolipoprotein E (APOE4) in the risk of developing $\mathrm{AD}$. Age and genetic background are the strongest known risk factors for AD (Blennow et al., 2006). The APOE4 allele, the major genetic susceptibility factor for late-onset $\mathrm{AD}$, present in $\sim 15 \%$ of the population (Strittmatter and Roses, 1996), confers dramatically increased risk in a gene dosedependent manner for the development of $\mathrm{AD}$ with an earlier age of onset (Corder et al., 1993). Other isoforms of APOE are con- 
Table 1. Demographic comparison of ApoE4+ and ApoE4- participants

\begin{tabular}{lllllll}
\hline & & & & \multicolumn{2}{c}{ ApoE4-versus } \\
& $\begin{array}{l}\text { Total PIB }- \\
(\mathrm{N}=100)\end{array}$ & $\begin{array}{l}\text { ApoE4 }- \\
(\mathrm{N}=62)\end{array}$ & $\begin{array}{l}\text { ApoE4+ } \\
(\mathrm{N}=38)\end{array}$ & \multicolumn{2}{c}{ ApoE4+ } \\
\cline { 6 - 8 } & 72,28 & 43,19 & 29,9 & $0.57^{*}$ & 0.45 \\
\hline Gender (female, male) & $61.6(8.1)$ & $63.3(7.4)$ & $58.8(8.5)$ & 2.81 & 0.01 \\
Mean age (SD) (years) & $16.1(2.4)$ & $16.0(2.6)$ & $16.2(2.0)$ & -0.37 & 0.72 \\
Mean education (SD) (years) & $29.4(0.8)$ & $29.3(0.9)$ & $29.6(0.7)$ & -1.57 & 0.12 \\
Mean MMSE score (SD) & & & & & 0.06 \\
Mean MCBP score (SD) & $0.02(0.06)$ & $0.01(0.05)$ & $0.03(0.07)$ & -1.94 & \\
\hline
\end{tabular}

MMSE, Mini Mental State Examination. * $\chi^{2}$ test, $d f=1$.

sidered to be neutral (APOE3) or protective (APOE2) for AD risk (Corder et al., 1994). In a small set of young healthy APOE4carriers, compared with matched noncarriers, Filippini et al. (2009) found increased coactivation in retrosplenial, medial temporal, and medial prefrontal cortex by independent component analysis in resting-state fMRI. Using resting-state data, Fleisher et al. (2009) found that another small sample of APOE4- carriers aged 50-65 years had increased default-mode network connectivity compared with noncarriers. However, it was not known whether these participants had fibrillar amyloid plaques in addition to the APOE4 genotype. In the current study, we investigated the role of APOE4 genotype on DMN connectivity in subjects who were shown by PIB PET to be negative for fibrillar amyloid plaques, to determine the effect of genotype on resting state connectivity, independent of amyloid plaque effects.

\section{Materials and Methods}

Participants were community-living volunteers enrolled in longitudinal studies of memory and aging at the Knight Alzheimer's Disease Research Center at Washington University. Individuals had no cognitive impairment as assessed by the Clinical Dementia Rating (CDR) (Morris, 1993) scale. These cognitively normal participants $($ all $C D R=0)$ also participated in PIB PET imaging to identify subjects $(n=100)$ with minimal $\mathrm{A} \beta$ deposition (PIB-), defined as mean cortical binding potential $(\mathrm{MCBP})<0.18$ (Mintun et al., 2006; Sheline et al., 2010) (mean $\mathrm{MCBP}=0.02$ in the current sample) (see Results for more details on demographics). Detailed information on PIB PET imaging and analysis has been reported previously (Mintun et al., 2006). Briefly, a $60 \mathrm{~min}$ dynamic PET scan was obtained after injection of $\sim 12 \mathrm{mCi}$ of $\left[{ }^{11} \mathrm{C}\right] \mathrm{PIB}$. Time activity curves derived from specific MRI regions of interest (ROI) were used to calculate the MCBP (Mintun et al., 2006).

Genotyping. DNA was extracted from peripheral blood samples using standard procedures. APOE genotyping was performed as previously described (Morris et al., 2010). Because the number of subjects with the APOE2 allele was small, they were combined as part of the APOE4- if the genotype was $2 / 2$ or $2 / 3$. If the genotype was $2 / 4$ they were counted as APOE4+.

CDR 0 cognitively normal participants who were PIB-, defined as MCBP $<0.18$ (Mintun et al., 2006; Sheline et al., 2010) ( mean $=0.02$; $\mathrm{SD}=0.06$ ) were categorized into two groups based on the presence or absence of the APOE4 allele. If the participant had at least one APOE4 allele they were categorized as APOE4+ and if they had no APOE4 allele they were categorized as APOE4-. We combined the APOE 4/4 genotype $(n=4)$ with APOE $3 / 4(n=29)$ and APOE $2 / 4(n=5)$ for a total of 38 individuals who were APOE4 carriers $(\mathrm{APOE} 4+)$. These subjects were compared with the remaining 62 subjects with APOE $3 / 3(n=50)$, APOE $2 / 3(n=10)$, and APOE $2 / 2(n=2)$ (APOE4-) genotype. Demographic comparison of APOE4+ versus APOE4- individuals is shown in Table 1. All of the connectivity analyses were regressed on APOE4, adjusting for age, education, gender, and MCBP. The main effect of covariates was not statistically significant $(p>0.05)$.

fMRI scanning methods. These methods have been described previously (Sheline et al., 2010). Subjects were scanned with their eyes closed in a $3 \mathrm{~T}$ Siemens Trio scanner and functional images were collected in runs using a gradient echo sequence [echo time $=27 \mathrm{~ms}$; repetition time $(\mathrm{TR})=384 \mathrm{~ms}$; field of view $=256 \mathrm{~mm}$; flip angle $\left.=90^{\circ}\right]$ sensitive to blood oxygenation level-dependent (BOLD) contrast ( $\mathrm{T} 2^{\star}$ weighting). Thirty-six contiguous, $4.0-\mathrm{mm}$-thick slices were acquired parallel to the anterior-posterior commissure plane $(4.0 \mathrm{~mm}$ approximately isotropic voxels), providing complete brain coverage. Two fMRI runs included 164 volumes each, continuously acquired at a TR of $2.2 \mathrm{~s}$ ( $\sim 6 \mathrm{~min}$ each). The fMRI data were corrected for head motion, transformed to a common atlas space, and blurred with a $6 \mathrm{~mm}$ full-width half-maximum Gaussian filter.

Functional connectivity analysis of resting-state activity. We used standard methods for the functional connectivity analysis (Vincent et al., 2006; Fox and Raichle, 2007; Fox et al., 2009). BOLD volumetric time series were concatenated and preprocessed, including temporal filtering, retaining frequencies up to $0.1 \mathrm{~Hz}$. Spurious variance was reduced by regression of several nuisance variables, including head motion parameters, and the signal averaged over the whole brain (Fox et al., 2009). There were no group differences in quality assurance measures, e.g., head movement.

Creation of a voxel restriction mask based on prior work. This procedure generated a mask that was used to enhance the likelihood of observing significant $A P O E 4+$ versus APOE4- effects in the current study (supplemental materials, available at www.jneurosci.org).

APOE4+ versus APOE4- precuneus functional connectivity comparison. Individual correlation maps were produced by extracting the BOLD time course from the bilateral precuneus seed region $( \pm 7,-60,21)$ used in previous analysis of $\mathrm{AD}$ versus control participants (Sheline et al., 2010). Correlation maps were computed using the Pearson productmoment formula and applied to the voxelwise BOLD time course. Individual subjects' correlation maps were Fisher $z$ transformed and entered into a voxelwise random-effects analysis (two-sided unequal variance) of the APOE4+ versus APOE4- effect. The resulting $t$ map was converted to equiprobable $\mathrm{Z}$ scores, masked by a priori hypothesized ROIs (see above) to retain voxels most likely to show a group difference, and then subjected to peak search. Peak search retained voxels with a significance of $p \leq 0.01$, not corrected for multiple comparisons, with a minimum of 10 contiguous voxels, leaving 14 regions of interest. For each participant, time series were extracted from the identified regions and the precuneusROI correlation coefficients were computed. Statistical significance of group differences was assessed on the basis of $t$ tests on the precuneusROI correlations (Table 2). In addition, Bonferroni correction for multiple comparisons on the 14 original regions was conducted, yielding nine significant ROIs following correction (Table 2).

\section{Results}

APOE 4+ versus APOE 4- connectivity using a priori regions defined in $\mathrm{AD}$

The regions of difference are shown in Table 2. Regions $(n=9)$ surviving a Bonferroni multiple-comparisons correction $(n=$ 14) are also indicated in Table 2 and shown in Figure 1.

In Figure 1, the hue of the region indicates the sign of the measured group difference in precuneus correlation (blue, $\mathrm{r}_{\mathrm{APOE} 4+}<\mathrm{r}_{\mathrm{APOE} 4-} ;$ red, $\left.\mathrm{r}_{\mathrm{APOE} 4+}>\mathrm{r}_{\mathrm{APOE} 4-}\right)$. Regions with positive APOE4+ versus APOE4- connectivity group differences included regions with increased positive correlation [medial prefrontal cortex (MPFC) and BA10 (frontal pole)] and regions with anticorrelation in APOE4- that had positive correlation in APOE4+ [caudal orbital cortex and dorsal occipital cortex (BA19)].

Regions with negative APOE4 + versus APOE4 - connectivity group differences included regions of decreased positive correlation (left hippocampus, left parahippocampus, middle temporal cortex, and BA20), a region with increased anti-correlation [dorsal anterior cingulate $(\mathrm{AC})]$, and regions with positive correlations in $A P O E 4$ - that had negative correlation in APOE4+ [right gyrus rectus, right hippocampus, and left superior temporal gyrus/frontoparietal operculum (BA22)]. These differences are also shown graphically in Figure 2. 
Table 2. Regions in ApoE4+ versus ApoE4 - participants with significantly different connectivity of the bilateral precuneus to a priori regions

\begin{tabular}{|c|c|c|c|c|}
\hline \multirow[b]{2}{*}{ Regions } & \multirow[b]{2}{*}{$(x, y, z)$} & \multirow[b]{2}{*}{ Voxels } & \multicolumn{2}{|l|}{$p$ Value } \\
\hline & & & Uncorrected & Corrected \\
\hline Left superior temporal gyrus/fronto-parietal operculum (BA22) & $-37,-40,+9$ & 21 & 0.001 & $0.009^{*}$ \\
\hline Right superior temporal gyrus/fronto-parietal operculum (BA22) & $+51,-3,+8$ & 13 & 0.006 & 0.082 \\
\hline Dorsal anterior cingulate (BA32) & $-14,+18,+30$ & 27 & $<0.001$ & $0.006^{*}$ \\
\hline Caudal orbital cortex & $-13,+24,-16$ & 32 & $<0.001$ & $0.004^{*}$ \\
\hline Pregenual anterior cingulate (BA24)/striatum & $-02,+24,+02$ & 19 & 0.013 & 0.179 \\
\hline Right hippocampus & $+19,-19,-6$ & 14 & $<0.001$ & $0.004^{*}$ \\
\hline Medial prefrontal cortex (frontal pole) (BA10) & $+16,+53,+18$ & 15 & 0.001 & $0.020^{*}$ \\
\hline Inferior orbital cortex (BA11) & $+7,+58,-21$ & 13 & 0.004 & 0.054 \\
\hline Gyrus rectus (BA11) & $+4,+23,-21$ & 11 & 0.001 & $0.014^{*}$ \\
\hline Hypothalamus & $0,0,-9$ & 15 & 0.012 & 0.168 \\
\hline Dorsal occipital cortex (BA19) & $0,-89,+40$ & 27 & 0.002 & $0.029^{*}$ \\
\hline
\end{tabular}

Regions were derived from comparisons of a priori areas shown in supplemental Figure 1 (available at www.jneurosci.org as supplemental material) and are shown in Figures 1 and 2. Listed are the Talairach coordinates, voxel number (size) and significance level for each region. $p$ values denote significant differences in connectivity between APOE $4+$ and APOE $4-$ groups. *Significant at $p \leq 0.05$ after Bonferroni correction for multiple comparisons.
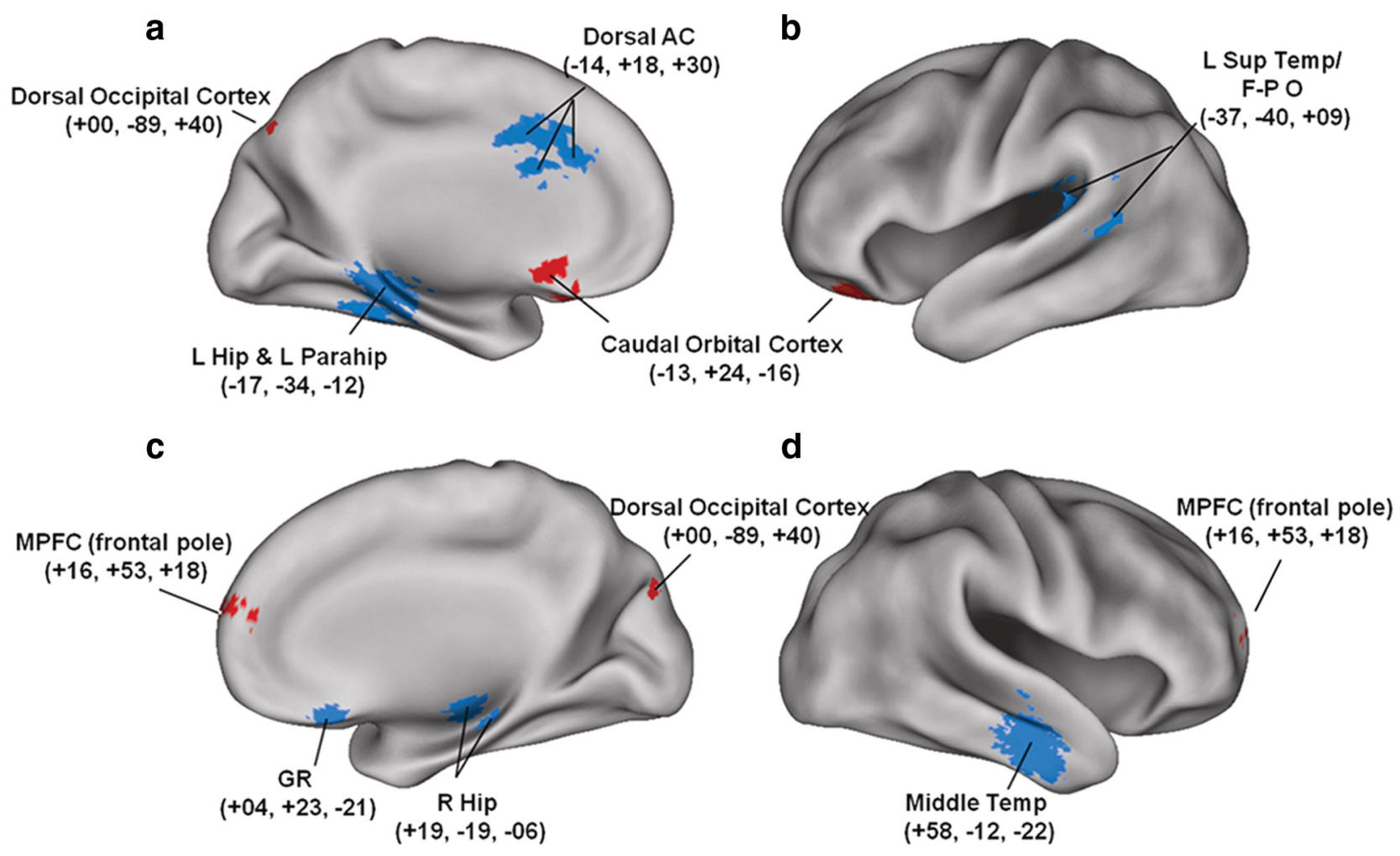

Figure 1. Altered functional connectivity of the precuneus in cognitively normal PIB - APOE4 + versus APOE4 - participants. $\boldsymbol{a}-\boldsymbol{d}$, Medial and lateral sagittal sections identifying statistically significant regional differences in functional connectivity of the precuneus between APOE4+ and APOE4 - cognitively normal individuals previously determined by PIB PET studies to be PIB - . L, Left; R, right; Hip, hippocampus; Parahip, parahippocampus; Temp, temporal cortex; GR, gyrus rectus; Sup Temp/F-P 0, superior temporal gyrus/frontoparietal operculum.

In addition, we conducted post hoc analyses limiting the sample to the 70 of 100 participants with CSF levels of $A \beta_{42}>500$ $\mathrm{pg} / \mathrm{ml}$, the cutoff in our dataset for abnormal CSF A $\beta_{42}$ (Morris et al., 2010) using the Innotest ELISA (Immogenetics). We found that of the nine Bonferroni multiple-comparison corrected regions identified in Table 2 and Figure 1, five remained significant in the comparison of ApoE4 + versus ApoE4 - group connectivity differences when limited to those participants with CSF levels of $\mathrm{A} \beta_{42}>500 \mathrm{pg} / \mathrm{ml}$, another biomarker used to determine preclinical AD. These regions were left hippocampus, left parahippocampus, dorsal anterior cingulate, dorsal occipital cortex, and middle temporal cortex.
For exploratory APOE $4+$ versus APOE $4-$ connectivity in whole brain analyses, see supplemental materials, available at www.jneurosci.org.

\section{Discussion}

In the current study, participants carrying an APOE4 allele had clear-cut abnormalities in precuneus resting-state functional connectivity in the absence of any cognitive impairment, and in the absence of fibrillar cerebral $\mathrm{A} \beta$ deposits detectable by PET PIB imaging. This result suggests that genetic influences altered functional connectivity within the DMN before the onset of clinical disease. We have previously shown an APOE4 dose- 


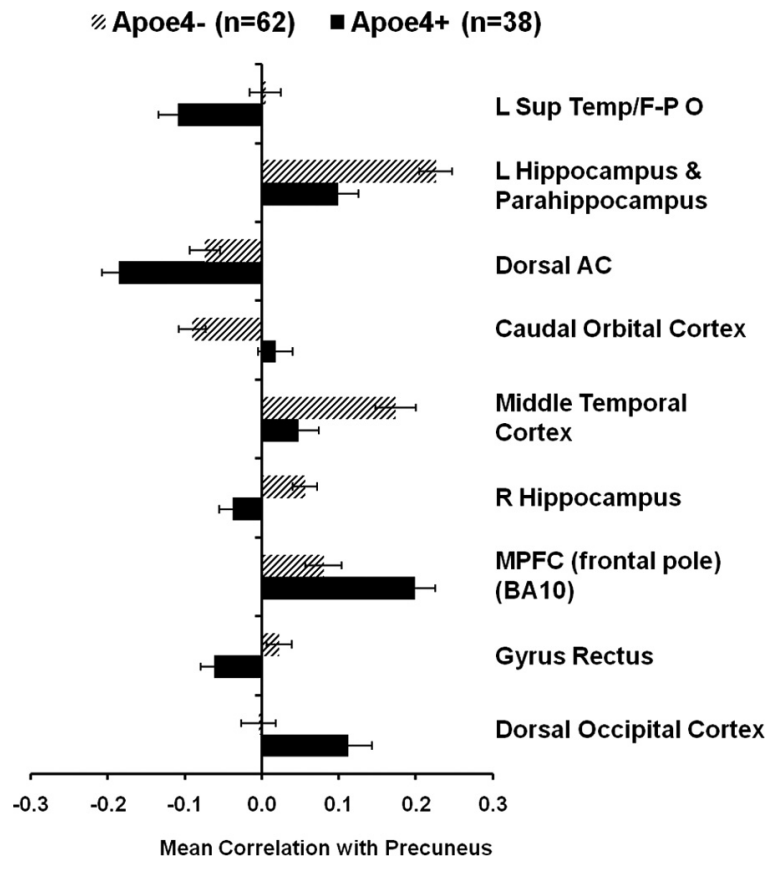

Figure 2. Altered functional connectivity of the precuneus in cognitively normal PIBAPOE4 + versus APOE4 - participants. The graph compares regional correlation magnitudes for APOE4 + and APOE4 - individuals for regions shown in Figure 1. L, Left; R, right; Sup Temp/F-P 0, superior temporal gyrus/frontoparietal operculum.

dependent increase in MCBP values, with an odds ratio of 5.04 (95\% CI, 2.53-10.2) for likelihood of high MCBP in individuals with an APOE4 allele (Morris et al., 2010). Effects of the APOE4 allele have been demonstrated in amyloid imaging studies in individuals with moderate AD (Drzezga et al., 2009) and in cognitively normal individuals (Reiman et al., 2009; Small et al., 2009; Morris et al., 2010). In addition, in AD, the APOE4 allele has been shown to have greater impairment on memory retention, whereas noncarriers were more impaired on tests of working memory, executive function, and language (Wolk et al., 2010). We observed, in the absence of fibrillar $\mathrm{A} \beta$ plaque deposition, regional alterations in $\mathrm{fcMRI}$ in many of the same brain areas that show disrupted connectivity in PIB + individuals and in frank $\mathrm{AD}$ (Sheline et al., 2010). Interestingly, most of the regions identified with effects of APOE4, in particular, bilateral hippocampus and left parahippocampus, had decreased connectivity with the precuneus. This result stands in contrast to two small restingstate studies of APOE4 effects on DMN finding increased connectivity (Filippini et al., 2009; Fleisher et al., 2009). There was no effect of regression analysis on results using CSF A $\beta_{42}$ as a covariate. Further, in post hoc analyses of the subset of 70 participants screened to exclude those with abnormally low CSF A $\beta_{42}$ levels, we found similar patterns of abnormal functional connectivity, although there were less regions that survived, perhaps due to a reduced sample size ( $n=70$ vs 100 ) or to an effect of CSF A $\beta_{42}$ levels. These results suggest that mechanisms resulting in increased neuronal toxicity might already be in place before lowered $\mathrm{A} \beta_{42}$ CSF levels.

Altered functional connectivity between the posterior and anterior portions of the default-mode network has been described in $\mathrm{AD}$ (Greicius and Menon, 2004) and in preclinical $\mathrm{A} \beta$ plaque deposition (Hedden et al., 2009; Sheline et al., 2010). We found these same regional differences in precuneus connectivity in the current study as seen in $\mathrm{AD}$, with the exception of increased anticorrelation with dorsal AC and left superior temporal cortex/ frontal-parietal operculum. In addition to differences predicted from a priori regions in $\mathrm{AD}$, we also found additional regions with alterations in precuneus connectivity in APOE4 allele carriers in whole-brain exploratory analyses, indicating that altered resting-state functional organization already had occurred in normal aging in the presence of APOE4 genotype but in the absence of deposition of $A \beta$ plaques. However, interpretive caution is warranted since differences were observed in exploratory analyses and require replication in an independent sample. One interesting discrepancy was observed between the increased connectivity with MPFC in the a priori analysis versus decreased connectivity with MPFC in whole-brain analysis. We note, however, that the MPFC region from whole-brain analysis was considerably more caudal $(z=-12)$ than the a priori region $(z=$ 18 ), and as such has different medial network connections (Carmichael and Price, 1996). The significance of these regions of altered connectivity remains to be determined but, as discussed below, may reflect activity-dependent APOE4 effects on $\mathrm{A} \beta$ amyloid deposition and on neuronal stability. We note that most of the alterations involved decreased connectivity with the precuneus and most of these additional regions were also in the DMN, highlighting the importance of early damage to the DMN as a potential manifestation of preclinical AD.

Regions in the DMN (Raichle et al., 2001) exhibit structural and functional connectivity that converge on the precuneus and posterior cingulate (Hagmann et al., 2008). During attentiondemanding tasks, there are decreases in activity, correlated with task difficulty and successful memory encoding (Lustig et al., 2003; Daselaar et al., 2004). Patients with mild AD (Lustig et al., 2003), mild cognitive impairment (Petrella et al., 2007), and preclinical A $\beta$ amyloid deposition (Sperling et al., 2009), however, have demonstrated a paradoxical reversal in this pattern, with increased task-related fMRI activity in areas of $\mathrm{A} \beta$ deposition, implicating amyloid deposition in dysfunctional brain networks supporting memory formation. Further, we have shown that the regions with earliest $\mathrm{A} \beta$ deposition lie within the $\mathrm{DMN}$ and also have the highest resting metabolic activity (Buckner et al., 2005). Since DMN regions are known to have the highest resting-state metabolic activity, it may be that those APOE4-mediated mechanisms that depend on metabolic activity, such as decreased transport and damage to astroglia, occur at a higher rate within the DMN regions, and that these effects predate deposition of $\mathrm{A} \beta$ plaques.

The effect of APOE4 appears to operate through both $\mathrm{A} \beta$ metabolism effects and non-A $\beta$ effects. Clear evidence supports $\mathrm{A} \beta$ metabolism effects as a major mechanism for $A P O E$ genotype influence on AD. Histopathological studies demonstrated a positive correlation between APOE 4 dose and $\mathrm{A} \beta$ plaque density (Rebeck et al., 1993) and increased neuritic plaques (Tiraboschi et al., 2004). Further, cognitively normal subjects had an APOE4 dose-dependent increase in fibrillar $\mathrm{A} \beta$ plaque burden, detected by amyloid imaging (Reiman et al., 2009). Studies have also found that APOE4 was more effective than APOE3 at increasing $\mathrm{A} \beta 40$ aggregation (Wisniewski et al., 1994). In addition to effects on fibrillogenesis, there is evidence that APOE4 alters both brain transport and metabolism of $A \beta$. A $\beta$ in complex with APOE2 and APOE3 is cleared more rapidly from the brain than $A \beta$ complexed to APOE4 (Deane et al., 2008).

Additional evidence suggests that APOE4 also plays a role in non-A $\beta$-mediated mechanisms (Zhong and Weisgraber, 2009), resulting in decreased neuronal stability and plasticity. Among critical differences are a lower stability of the APOE4 isoform with greater propensity to form toxic aggregates (Hatters et al., 
2006); lower E4 affinity for small lipoproteins, resulting in less effective transport of lipids required for neuronal synaptogenesis, maintenance, and repair (Dong et al., 1994); and increased cleavage of APOE4 by neuronal protease, forming fragments that escape the secretory pathway and lead to neuronal death (Harris et al., 2003). An isoform-specific effect of APOE on neurite outgrowth has been reported and, although inconclusive regarding the effects of APOE on neuronal processes (Kim et al., 2009), it is possible that astrocytes contribute more directly to the association of APOE4 and AD than had previously been thought. An interesting phenomenon involves a switch in APOE4 expression. Normally APOE is only expressed in astrocytes and other glial cells. However, under stressful conditions, neurons also express APOE (Xu et al., 2006). Further, APOE4 produced within neurons is subject to cleavage by neuronal protease, resulting in toxic fragments. These toxic fragments have been observed in $\mathrm{AD}$ patients and in transgenic mice expressing APOE4 in neurons (Zhong et al., 2009). An additional pathological mechanism involves APOE4 alteration of the ability of astrocytes to facilitate neuronal function: Over most of a lifetime, although compromised, astrocyte function is sufficient in non-stressed conditions (Zhong et al., 2009). However, in the presence of later-life stressors such as ischemia, oxidative stress, and $\mathrm{A} \beta$ toxicity, astrocyte support can become less effective. Thus, APOE4 can contribute to $\mathrm{AD}$ pathology by acting through both $\mathrm{A} \beta$-dependent and $\mathrm{A} \beta$ independent pathways.

Although many APOE4 changes in our study occurred in the same areas that are affected in $\mathrm{AD}$ and these effects could be interpreted as very early manifestations of $\mathrm{AD}$, our exploratory whole-brain analyses revealed other regions with altered functional connectivity and may represent APOE4 effects that are independent of $\mathrm{AD}$ pathology. For example, APOE4 genotype could be associated with a genetically altered functional connectivity, some portion of which is unassociated with AD. Alternatively, these changes could have pathological significance and be associated with toxic APOE4 allele effects independent of ADrelated toxicity. The current finding that APOE4 allele carriers had alterations in precuneus resting-state functional connectivity with other important DMN regions highlights the importance of integrity of the DMN (Raichle et al., 2001). Indeed, a role for an APOE4 effect mediated through metabolic demands is suggested by the distribution of preclinical fibrillar $A \beta$ falling within DMN regions (Shulman et al., 1997; Raichle et al., 2001; Buckner et al., 2005), which have very high resting metabolic demands. Evidence from mouse models (Cirrito et al., 2005) suggests neuronal activity-dependent increase in production of $A \beta$. In APOE4 allele carriers, who have chronic deficient astrocyte support, the vulnerability of DMN regions to neuronal damage could be triggered in the face of additional burden, such as ischemia or oxidative stress. This could help to explain the greater risk and earlier age of onset of dementia in APOE4 carriers. Longitudinal studies will be important to determine the time course of any alterations in resting-state functional connectivity, both those that appear independent of fibrillogenesis and, particularly, those that appear to progress to neuronal dysfunction and damage, to help plan the optimal timing for potential interventional studies in individuals at risk for symptomatic AD.

\section{References}

Blennow K, de Leon MJ, Zetterberg H (2006) Alzheimer's disease. Lancet 368:387-403.

Buckner RL, Snyder AZ, Shannon BJ, LaRossa G, Sachs R, Fotenos AF, Sheline YI, Klunk WE, Mathis CA, Morris JC, Mintun MA (2005) Molecular, structural, and functional characterization of Alzheimer's disease: evidence for a relationship between default activity, amyloid, and memory. J Neurosci 25:7709-7717.

Cairns NJ, Ikonomovic MD, Benzinger T, Storandt M, Fagan AM, Shah A, Reinwald LT, Carter D, Felton A, Holtzman DM, Mintun MA, Klunk WE, Morris JC (2009) Absence of Pittsburgh Compound B detection of cerebral amyloid beta in a patient with clinical, cognitive, and cerebrospinal fluid markers of Alzheimer disease. Arch Neurol 66:1557-1562.

Carmichael ST, Price JL (1996) Connectional networks within the orbital and medial prefrontal cortex of macaque monkeys. J Comp Neurol 371:179-207.

Cirrito JR, Deane R, Fagan AM, Spinner ML, Parsadanian M, Finn MB, Jiang H, Prior JL, Sagare A, Bales KR, Paul SM, Zlokovic BV, Piwnica-Worms D, Holtzman DM (2005) P-glycoprotein deficiency at the blood-brain barrier increases amyloid-beta deposition in an Alzheimer disease mouse model. J Clin Invest 115:3285-3290.

Corder EH, Saunders AM, Strittmatter WJ, Schmechel DE, Gaskell PC, Small GW, Roses AD, Haines JL, Pericak-Vance MA (1993) Gene dose of apolipoprotein E type 4 allele and the risk of Alzheimer's disease in late onset families. Science 261:921-923.

Corder EH, Saunders AM, Risch NJ, Strittmatter WJ, Schmechel DE, Gaskell PC Jr, Rimmler JB, Locke PA, Conneally PM, Schmader KE (1994) Protective effect of apolipoprotein E type 2 allele for late onset Alzheimer disease. Nat Genet 7:180-184.

Daselaar SM, Prince SE, Cabeza R (2004) When less means more: deactivations during encoding that predict subsequent memory. Neuroimage 23:921-927.

Deane R, Sagare A, Hamm K, Parisi M, Lane S, Finn MB, Holtzman DM, Zlokovic BV (2008) apoE isoform-specific disruption of amyloid beta peptide clearance from mouse brain. J Clin Invest 118:4002-4013.

Dong LM, Wilson C, Wardell MR, Simmons T, Mahley RW, Weisgraber KH, Agard DA (1994) (1994) Human apolipoprotein E: role of arginine 61 in mediating the lipoprotein preferences of the $\mathrm{E} 3$ and $\mathrm{E} 4$ isoforms. J Biol Chem 269:22358-22365.

Drzezga A, Grimmer T, Henriksen G, Mühlau M, Perneczky R, Miederer I, Praus C, Sorg C, Wohlschläger A, Riemenschneider M, Wester HJ, Foerstl H, Schwaiger M, Kurz A (2009) Effect of APOE genotype on amyloid plaque load and gray matter volume in Alzheimer disease. Neurology 72:1487-1494.

Filippini N, MacIntosh BJ, Hough MG, Goodwin GM, Frisoni GB, Smith SM, Matthews PM, Beckmann CF, Mackay CE (2009) Distinct patterns of brain activity in young carriers of the APOE-epsilon4 allele. Proc Natl Acad Sci U S A 106:7209-7214.

Fleisher AS, Sherzai A, Taylor C, Langbaum JB, Chen K, Buxton RB (2009) Resting-state BOLD networks versus task-associated functional MRI for distinguishing Alzheimer's disease risk groups. Neuroimage 47:16781690.

Fox MD, Raichle ME (2007) Spontaneous fluctuations in brain activity observed with functional magnetic resonance imaging. Nat Rev Neurosci 8:700-711.

Fox MD, Zhang D, Snyder AZ, Raichle ME (2009) The global signal and observed anticorrelated resting state brain networks. J Neurophysiol 101:3270-3283.

Greicius MD, Menon V (2004) Default-mode activity during a passive sensory task: uncoupled from deactivation but impacting activation. J Cogn Neurosci 16:1484-1492.

Hagmann P, Cammoun L, Gigandet X, Meuli R, Honey CJ, Wedeen VJ, Sporns O (2008) Mapping the structural core of human cerebral cortex. PLoS Biol 6:e159.

Harris FM, Brecht WJ, Xu Q, Tesseur I, Kekonius L, Wyss-Coray T, Fish JD, Masliah E, Hopkins PC, Scearce-Levie K, Weisgraber KH, Mucke L, Mahley RW, Huang Y (2003) Carboxyl-terminal-truncated apolipoprotein E4 causes Alzheimer's disease-like neurodegeneration and behavioral deficits in transgenic mice. Proc Natl Acad Sci U S A 100:10966-10971.

Hatters DM, Zhong N, Rutenber E, Weisgraber KH (2006) Amino-terminal domain stability mediates apolipoprotein $\mathrm{E}$ aggregation into neurotoxic fibrils. J Mol Biol 361:932-944.

Hedden T, Van Dijk KR, Becker JA, Mehta A, Sperling RA, Johnson KA, Buckner RL (2009) Disruption of functional connectivity in clinically normal older adults harboring amyloid burden. J Neurosci 29:1268612694. 
Kim J, Basak JM, Holtzman DM (2009) The role of apolipoprotein E in Alzheimer's disease. Neuron 63:287-303.

Lustig C, Snyder AZ, Bhakta M, O’Brien KC, McAvoy M, Raichle ME, Morris JC, Buckner RL (2003) Functional deactivations: change with age and dementia of the Alzheimer type. Proc Natl Acad Sci U S A 100:1450414509.

Mintun MA, Larossa GN, Sheline YI, Dence CS, Lee SY, Mach RH, Klunk WE, Mathis CA, DeKosky ST, Morris JC (2006) [11C]PIB in a nondemented population: potential antecedent marker of Alzheimer disease. Neurology $67: 446-452$.

Morris JC (1993) The Clinical Dementia Rating (CDR): current version and scoring rules. Neurology 43:2412-2414.

Morris JC, Roe CM, Xiong C, Fagan AM, Goate AM, Holtzman DM, Mintun MA (2010) APOE predicts amyloid-beta but not tau Alzheimer pathology in cognitively normal aging. Ann Neurol 67:122-131.

Petrella JR, Wang L, Krishnan S, Slavin MJ, Prince SE, Tran TT, Doraiswamy PM (2007) Cortical deactivation in mild cognitive impairment: highfield-strength functional MR imaging. Radiology 245:224-235.

Price JL, Morris JC (1999) Tangles and plaques in nondemented aging and "preclinical“" Alzheimer's disease. Ann Neurol 45:358-368.

Raichle ME, MacLeod AM, Snyder AZ, Powers WJ, Gusnard DA, Shulman GL (2001) A default mode of brain function. Proc Natl Acad Sci U S A 98:676-682.

Rebeck GW, Reiter JS, Strickland DK, Hyman BT (1993) Apolipoprotein E in sporadic Alzheimer's disease: allelic variation and receptor interactions. Neuron 11:575-580.

Reiman EM, Chen K, Liu X, Bandy D, Yu M, Lee W, Ayutyanont N, Keppler J, Reeder SA, Langbaum JB, Alexander GE, Klunk WE, Mathis CA, Price JC, Aizenstein HJ, DeKosky ST, Caselli RJ (2009) Fibrillar amyloid-beta burden in cognitively normal people at 3 levels of genetic risk for Alzheimer's disease. Proc Natl Acad Sci U S A 106:6820-6825.

Sheline YI, Raichle ME, Snyder AZ, Morris JC, Head D, Wang S, Mintun MA (2010) Amyloid plaques disrupt resting state default mode network connectivity in cognitively normal elderly. Biol Psychiatry 67:584-587.

Shulman GL, Fiez JA, Corbetta M, Buckner RL, Miezin FM, Riachle ME,
Petersen SE (1997) Common blood flow changes across visual tasks. II. Decreases in cerebral cortex. J Cogn Neurosci 9:648-663.

Small GW, Siddarth P, Burggren AC, Kepe V, Ercoli LM, Miller KJ, Lavretsky H, Thompson PM, Cole GM, Huang SC, Phelps ME, Bookheimer SY, Barrio JR (2009) Influence of cognitive status, age, and APOE-4 genetic risk on brain FDDNP positron-emission tomography imaging in persons without dementia. Arch Gen Psychiatry 66:81-87.

Sperling RA, Laviolette PS, O’Keefe K, O’Brien J, Rentz DM, Pihlajamaki M, Marshall G, Hyman BT, Selkoe DJ, Hedden T, Buckner RL, Becker JA, Johnson KA (2009) Amyloid deposition is associated with impaired default network function in older persons without dementia. Neuron 63:178-188.

Strittmatter WJ, Roses AD (1996) Apolipoprotein E and Alzheimer's disease. Annu Rev Neurosci 19:53-77.

Tiraboschi P, Hansen LA, Masliah E, Alford M, Thal LJ, Corey-Bloom J (2004) Impact of APOE genotype on neuropathologic and neurochemical markers of Alzheimer disease. Neurology 62:1977-1983.

Vincent JL, Snyder AZ, Fox MD, Shannon BJ, Andrews JR, Raichle ME, Buckner RL (2006) Coherent spontaneous activity identifies a hippocampal-parietal memory network. J Neurophysiol 96:3517-3531.

Wisniewski T, Castaño EM, Golabek A, Vogel T, Frangione B (1994) Acceleration of Alzheimer's fibril formation by apolipoprotein E in vitro. Am J Pathol 145:1030-1035.

Wolk DA, Dickerson BC, the Alzheimer's Disease Neuroimaging Initiative (2010) Apolipoprotein E (APOE) genotype has dissociable effects on memory and attentional-executive network function in Alzheimer's disease. Proc Natl Acad Sci U S A 107:10256-10261.

Xu Q, Bernardo A, Walker D, Kanegawa T, Mahley RW, Huang Y (2006) Profile and regulation of apolipoprotein E (ApoE) expression in the CNS in mice with targeting of green fluorescent protein gene to the ApoE locus. J Neurosci 26:4985-4994.

Zhong N, Weisgraber KH (2009) Understanding the basis for the association of apoE4 with Alzheimer's disease: opening the door for therapeutic approaches. Curr Alzheimer Res 6:415-418. 